\title{
Chaotic Particle Swarm Optimization Algorithm for Hub and Spoke Sys- tems with Congestion
}

\author{
Weiwei $\mathrm{Wu}^{*}$ and Hui Wang
}

Nanjing University of Aeronautics and Astronautics, Nanjing, Jiangsu, 210016, P.R. China

\begin{abstract}
Considering the hub airports are traffic transfer points, the congestion is easily happened. The cost caused by congestion will rise significantly. The hub-and-spoke airline network optimization model with congestion cost is designed. In the actual operations of airlines, such problem is difficult to be solved by using the classical optimization methods. This paper presents a Particle Swarm Optimization (PSO) algorithm. To improve the performance of standard PSO algorithm and avoid trapping into local excellent result, a chaos PSO algorithm of traffic volume multi-path assignment is presented. Empirical analysis shows that optimization design with congestion cost can avoid the excessive congestion phenomenon in the hub nodes. The proposed algorithm can solve the non-linear network optimization problem efficiently.
\end{abstract}

Keywords: Chaos, congestion cost, hub-and-spoke airline network, particle swarm optimization.

\section{INTRODUCTION}

Airline network layout has important strategic significance for the long term operation and market competitiveness of a company. In air transportation planning, the two most commonly used transport network layouts are point-topoint $(P P)$ and hub-and-spoke $(H S)$ structure. The disadvantage of $P P$ networks is that they cannot effectively use the economies of scale, and currently most researches mainly focus on $H S$ airline network design [1].

The literature [2-5] respectively present single allocation or multiple allocation mixed integer programming network design model, in which the goal is generally considered operation cost and construction cost. The solution is Lagrange relaxation algorithm, dual-ascent algorithm, heuristic based on tabu search and branch and bound method.

Grove and O'Kelly [6] simulates the operation of single allocation $H S$ system, and analyzes that aircraft delay is greatly influenced by the heavy flow in hub airports.

Some papers [7-12] have tackled the congestion effects restricting the amount of flow transiting through a hub by means of capacity constraints. However, in the study of network design, it is insufficient that we only consider that the flow of hub node cannot exceed its capacity limit, because with the traffic flow close to capacity, the airport begins to appear congestion and lower utilization of resources. So we must consider the congestion effect of hub airport. With the increasing traffic flow of hub airports, the congestion is increasing, operation cost will show non-linear increasing trend. The current airline network design model and its algorithm cannot effectively solve the congestion problem.
Based on the above analysis, this paper mainly studies on the $H S$ network optimization design, considering the congestion cost in the objective function. We can use heuristic algorithms to handle non-linear objective function, such as neural network algorithm, genetic algorithm and ant algorithm. However, the shortcomings of these methods are to solve with low efficiency and poor stability, which cannot ensure the optimization effect of solution.

The particle swarm algorithm has a few individual numbers, simple calculation, good robustness and parallel computing advantage. In order to avoid falling into local optimal solution, it can combine with chaos and particle swarm optimization [13]. Thus, this paper constructs a chaotic particle swarm optimization algorithm (CPSOA) of multi path traffic assignment, the solving result can avoid the heavy traffic flow of any one hub node exceeding its capacity restriction, which has effective shunt effect. Also, it guarantees the efficiency for solving large nonlinear network optimization problems.

\section{MODEL FORMULATION: HS NETWORK DESIGN WITH CONGESTION}

Considering hub airport is gathering the large amount of traffic flow, congestion occurs mainly in the hub airport. Elhedhli and $\mathrm{Hu}[14]$ has considered the costs of the congestion effects explicitly on the objective function. Using a convex cost function that increases exponentially as more flows go through the hubs.

$$
f(u)=a u^{b}
$$

where $u$ is the flow at a hub; $a$ and $b$ are positive constants with $b \geq 1$.

Using the model of the uncapacitated multiple allocation $p$ hub location problem (UMA $p$ HMP) [15], the flow through 

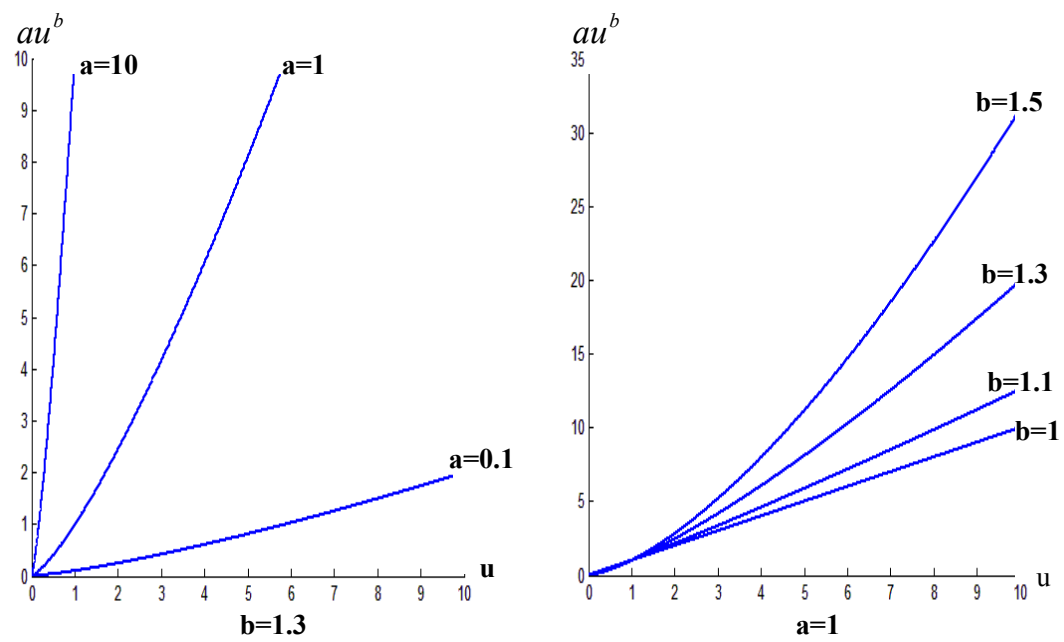

Fig. (1). The Congestion Cost Functions with Different Values of $a$ and $b$.

hub $k$ is $\sum_{r} \sum_{s>r} \sum_{m} W_{r s} X_{r s k m}$. So, the congestion cost at hub $k$ is $f(u)$.

$$
f(u)=a u^{b}=a\left(\sum_{r} \sum_{s>r} \sum_{m} W_{r s} X_{r s k m}\right)^{b}
$$

Fig. (1) plots the congestion cost functions for different values of the parameters $a$ and $b$.

Considering the congestion effect on network design, some improvement on UMA $p$ HMP model is presented. The congestion convex cost function is added into the objective function. The model is stated as follows:

$$
\begin{aligned}
& \min \quad \mathrm{Z}=\sum_{\mathrm{r}=1}^{\mathrm{n}} \sum_{\mathrm{s}=1}^{\mathrm{n}} \sum_{\mathrm{k}=1}^{\mathrm{n}} \sum_{\mathrm{m}=1}^{\mathrm{n}} \mathrm{W}_{\mathrm{rs}} \mathrm{C}_{\mathrm{rskm}} \mathrm{x}_{\mathrm{rskm}} \\
& +\sum_{\mathrm{k}} \mathrm{a}\left(\sum_{\mathrm{r}} \sum_{\mathrm{s}>r} \sum_{\mathrm{m}} \mathrm{W}_{\mathrm{rs}} \mathrm{X}_{\mathrm{rskm}}\right)^{\mathrm{b}} \\
& \text { s.t. } \sum_{k=1}^{n} y_{k}=p \\
& \sum_{k=1}^{n} \sum_{m=1}^{n} x_{r s k m}=1 ; r, s=1, \cdots, n \\
& \sum_{m=1}^{n} x_{r s k m} \leq y_{k} ; r, s, k=1, \cdots, n \\
& \sum_{k=1}^{n} x_{r s k m} \leq y_{m} ; r, s, m=1, \cdots, n \\
& y_{k} \in\{0,1\} ; k=1, \cdots, n ; \\
& 0 \leq x_{r s k m} \leq 1 ; r, s, k, m=1, \cdots, n
\end{aligned}
$$

The uncapacitated $H S$ network design is defined on a graph $G=(N, A)$, where $N$ is a set of nodes and $A$ is the set of routes $(r, s)$ (with origin node $r$ and destination node $s$ ). $w_{r s}$ be the flow from node $r$ to node $s$. We will generally assume that $w_{r s}=w_{s r}, r, s \in N$.

$x_{r s k m}$ be the fraction of flow from node $r$ to node $s$ that is routed via hubs at locations $k$ and $m$ in that order. If $k=m$, the variable $x_{r s k m}$ represents the one-hub-stop service through hub $k$.

$c_{r s k m}$ be the transportation cost per unit of flow from node $r$ to node $s$ routed via hubs $k$ and $m$. $k$ can be the same as $m$.

$y_{k}$ be a binary variable defined as follows: $y_{k}=\left\{\begin{array}{cc}1 & \text { if a hub is loacated at node } k \\ 0 & \text { otherwise }\end{array}\right.$.

The discount factor $\alpha$ represents the ecnomies of scale on the inter-hub connection, $0<\alpha<1$.

The objective function (1), $Z$ calculates the sum of congestion cost and operating cost. Constraint (2) states that the number of hubs to be located is $p$. Constraints (3) assure that the flow for every pair $r-s$ is routed via some hub pair. If only one hub is used, we have $k=m$. Constraints (4) and (5) express when a city is a non-hub city, there is no passengers transferring through it to other cities. Constraints (6) give the definition of decision variables.

The model is non-linear mixed integer planning problem. The papers $[14,16]$ solved it with Lagrangean heuristic algorithm and Benders algorithm. Considering the practicability of intelligent optimization algorithm, we use chaotic particle swarm optimization algorithm to solve this problem. CPSO algorithm has no limit for target function scale, which can be used to solve the nonlinear objective function model, and the complexity of the objective function has little impact on its convergence efficiency. 


\section{CHAOTIC PARTICLE SWARM OPTIMIZATION ALGORITHM}

\subsection{Algorithm Descriptions}

Dedicated to computational intelligence research, Kennedy [17] first proposed $P S O$ technique to replace the existing evolutionary techniques, such as genetic algorithm $(G A)$ [18]. The algorithm simulates bird foraging for purpose of cluster flight sharing mechanism to make the group behavior to achieve optimal information in a group. Chaos is a kind of nonlinear phenomenon widely existing in nature. It is chaotic, and its internal structure is very delicate, and it is sensitive to initial conditions, in a certain range according to fixed rules, which are not repeated traversal of all state. Chaos have the characteristics of randomness, ergodicity and regularity. The chaos properties can be used to conduct search optimization, in order to integrate into particle swarm algorithm, namely, so-called CPSOA.

For an optimization problem, the decision variable $\mathrm{X}$ is $\mathrm{n}$ dimensional variable $\mathrm{X}=\left[\mathrm{x}_{1}, \cdots, \mathrm{x}_{\mathrm{n}}\right]$, and the variables are called the particles. Assumed in the $n$-dimensional solution space, each particle has two state description, position and velocity, respectively, $\mathrm{X}_{\mathrm{i}}=\left(\mathrm{X}_{\mathrm{i} 1}, \mathrm{X}_{\mathrm{i} 2}, \cdots, \mathrm{X}_{\mathrm{in}}\right)$ and $\mathrm{V}_{\mathrm{i}}=$ $\left(\mathrm{V}_{\mathrm{i} 1}, \mathrm{~V}_{\mathrm{i} 2}, \cdots, \mathrm{V}_{\mathrm{in}}\right)$. The position $X_{i}$ represents the solution of problem.

The basic idea of CPSOA can be understood as: first initialized particle swarm and then in solution space, suppose in $t$ times, and the optimal solution of particle $i$ is $\operatorname{pbest}_{i}(t)$, and this is individual extremum, and the optimal solution for the whole particle swarm is gbest $(t)$, which is global extremum. When iterate over the $t+1$ times, its update expression is:

$$
\begin{aligned}
& \mathrm{V}_{\mathrm{i}}(\mathrm{t}+1)=\omega \mathrm{V}_{\mathrm{i}}(\mathrm{t})+\mathrm{c}_{1} \mathrm{r}_{1}\left[\text { pbest }_{\mathrm{i}}(\mathrm{t})-\mathrm{X}_{\mathrm{i}}(\mathrm{t})\right] \\
& +\mathrm{c}_{2} \mathrm{r}_{2}\left[\operatorname{gbest}(\mathrm{t})-\mathrm{X}_{\mathrm{i}}(\mathrm{t})\right] \\
& X_{i}(t+1)=X_{i}(t)+V_{i}(t+1)
\end{aligned}
$$

In the formula, $t$ represents the current iteration times, $c_{1}$, $c_{2}$ represent two learning factors, $c_{1}=c_{2}=2$, And two random variables $r_{1}, r_{2} \in(0,1)$. The inertia factor $\omega \in\left[\omega_{\max }, \omega_{\min }\right]$, $t_{\max }$ is the maximum number of iterations. In order to prevent the particle from leaving far away out of the searching space, the velocity of the particle is restricted in $\left[V_{\min }, V_{\max }\right]$, and the location of the particle also stays in permitted range, and finally the solution, gbest, is the global optimal.

According to the above mentioned basic particle swarm algorithm (BPSO), although it needs to confirm the parameters less, realizing process is simple, yet it is easy to trap into local optimum. Therefore chaotic dynamics is incorporated into the above $P S O$, the logistic reflection is defined as follows.

$$
\begin{aligned}
& \beta_{j}^{k+1}=\mu \beta_{j}^{k}\left(1-\beta_{j}^{k}\right), \quad k=1,2 \cdots, \\
& \beta_{j} \in(0,1), \quad \beta_{j} \neq 0.25,0.5,0.75
\end{aligned}
$$

\subsection{Algorithm Implementation}

The key point of PSO technique is to find a suitable expression to make the particle corresponding to the appropriate solution. One of the key problems of airline network optimization design is how to distribute the traffic flow in $O-D$ path. Thus, this paper considers constructing a path number dimensional space, and passengers need to select a feasible path, and each path value is the distributed flow of this path, namely, the particle corresponding vector $X_{i}$ represents a solution to the distribution of flow of each path.

The path flow $\mathrm{f}_{\mathrm{k}}^{\mathrm{rs}}$ and traffic demand $\mathrm{q}_{\mathrm{rs}}$ of any $O D$ path $(r, s)$ need to meet the flow conservation principle, thus, we need to make corresponding revision in algorithm, The CPSO algorithm steps are listed as below.

Step 1, (initialization):

(1) initialize $n, c_{1}, c_{2}, \omega$ maximum number of iterations.

(2) initialize each dimension of $X_{i}$ randomly between $\left[0, \mathrm{q}_{\mathrm{rs}}\right]$, that is, $\mathrm{f}_{\mathrm{k}}^{\mathrm{rs}}=\mathrm{q}_{\mathrm{rs}} \mathrm{c}_{\mathrm{R}}\left(\mathrm{c}_{\mathrm{R}} \in[0,1]\right)$. In order to ensure the constant value $\mathrm{q}_{\mathrm{rs}}$, it needs to conduct normalized processing of $\mathrm{f}_{\mathrm{k}}^{\mathrm{rs}}$.

$$
\overline{\mathrm{f}_{\mathrm{k}}^{\mathrm{rs}}}=\mathrm{q}_{\mathrm{rs}} \mathrm{f}_{\mathrm{k}}^{\mathrm{rs}} / \sum_{\mathrm{k}} \mathrm{f}_{\mathrm{k}}^{\mathrm{rs}}
$$

(3) Initialize each dimension of $V$ randomly between [1$\left.\mathrm{q}_{\mathrm{rs}}, \mathrm{q}_{\mathrm{rs}}-1\right]$ tion.

(4) Judge the fitness of all particles using objective func-

(5) Select the initial fitness values as the individual historical optimal solution $P_{i}$, then search for optimal solution within the swarm.

Step 2, Repeat until a stopping criterion or the maximal number of iteration is satisfied:

(1) For each particle, calculate particle swarm $V$ and $X$ from (7) and (8). When $V$ and $X$ exceed over the range, according to boundary value, and then normalized processing of $X$.

(2) Evaluate all the particles using objective function (1), when the current fitness function of a certain particle is better than its historical optimal fitness, the current fitness is remarked as historical optimal fitness, and the current position is the historical optimal location of this particle.

(3) Chaos optimized the optimal location $P_{g}$ of current particle swarm using Logistic reflection (9), in order to obtain chaos optimization result.

(4) Seeking for the current optimal solution in the swarm. If it is better than the history optimal solution, then update $P_{g}$. If the plurality of individual have optimal solutions, the randomly selected one is the current optimal solution.

During the solution process of CPSOA, paralled distribute the flow for each feasible path, and flow distribution as the solution of the corresponding particle will adjust the 
Table 1. 15 cities and their assigned number.

\begin{tabular}{|c|c|c|c|c|c|}
\hline Num. & Cities & Num. & Cities & Num. & Cities \\
\hline \hline 1 & Beijing & 6 & Hangzhou & 11 & Wuhan \\
\hline 2 & Changsha & 7 & Kunming & 12 & Urumqi \\
\hline 3 & Chengdu & 8 & Nanjing & 14 & Xiamen \\
\hline 4 & Guangzhou & 9 & Shanghai & 15 & Zhenzhou \\
\hline 5 & Haikou & 10 & Shenyang & & 15 \\
\hline
\end{tabular}

Table 2. Flow distribution ratio for 15 cities in China.

\begin{tabular}{|c|c|c|c|c|}
\hline \multirow{2}{*}{$(\boldsymbol{a}, \boldsymbol{b})$} & \multicolumn{3}{|c|}{ Flow Distribution Ratio } & \multirow{2}{*}{ Max/min } \\
\cline { 2 - 5 } & hub1 & hub2 & hub3 & 1.89 \\
\hline \hline$a=0$ & 42.8 & 22.7 & 34.8 & 1.43 \\
\hline$(1,1.3)$ & 39 & 27.2 & 33.0 & 1.27 \\
\hline$(1,1.5)$ & 37.5 & 29.5 & 32.6 & 1.09 \\
\hline$(1,2.0)$ & 35.2 & 32.2 & & \multirow{2}{*}{} \\
\hline
\end{tabular}

traffic on the path using the historical experience of individual and groups, thus gradually close to optimal position, so as to obtain the optimal result.

\section{COMPUTATIONAL EXPERIMENTS}

$H S$ topologies are an important class of network design that take full advantage of economies of scale on inter-hub connections. Hub location is very important and some airports having important region advantage become resources that airline companies use to compete. Considering the ranking order of cities, passenger traffic, geographical advantages and requirements of the Civil Aviation Administration of China, we can choose Beijing, Shanghai and Guangzhou as hub airports.

According to the airlines operational reports, the route cost is related to segment distance. In this computational example, we consider the route cost is the route distance corrected by empirical coefficient (airlines provided the cost of aircraft type operating the different routes). The passenger traffic between each pair of $O D$ is obtained from the Civil Aviation Statistical Yearbook 2009. Suppose that an airline has established bases in Beijing, Shanghai and Guangzhou and prepares to construct HS network with 15 cities (See Table 1).

The instance has been solved without congestion costs and then using $b=\{1.3,1.5,2.0\}$. The flow distribution ratio of three fixed hubs are shown in Table 2.

In Table 2, the results of the first row don't consider the congestion effects. From the second column to the fourth column, the percentage of the total demand is calculated in each installed hub. The last column is the flow imbalance ratio, that is the ratio of the largest over the lowest percentage hub flow. For instance, on row one, the flow imbalance is taken as $\frac{42.8}{22.7}=1.89$. namely, the large flow imbalance would happen when no congestion effects are assumed. When the congestion cost is considered, the sorting of flow distribution of three hub airports is not varied, but the flow imbalance ratio is decreased. For instance, $b=2$, the flow imbalance ratio tends to its minimum value of 1 . This can explained by the interference of congestion cost, the flow distribution of paths are very different from that of the uncapacitated model.

Figs. (2) and (3) are respectively flow distribution results of uncapacitated HS network and HS network with congestion cost. Although the two situations choose the same hub airports, their flow distribution path are different. In Fig. (3), the number of routes of network increases, the flow of $O D$ is no longer simply distributed with the shortest path, such as, the $O D$ flow from Xi'an to Nanjing, according to the shortest path, would select path as Xi'an-Beijing-Shanghai-Nanjing. Considering the congestion effect in hub airports, some passenger flow would choose Xi'an-Beijing-Nanjing, some other passenger flow would choose Xi'an-Shanghai-Nanjing. The $O D$ flow from Shenyang to Shanghai, the shortest path is Shenyang-Beijing-Shanghai, considering the congestion cost in hub airports, some traffic flow would choose direct flight from Shenyang to Shanghai.

Table 3 represents the flow distribution of some paths with three groups of congestion parameters. The paths are randomly selected, and the number of each node corresponds with the city number in Table $\mathbf{1}$. As $a$ and $b$ change, the distributed flow values of the same path are also different. The last three columns show that the flow distribution value of one path according to variations in $a$ and $b$, and 0 represents no flow distribution of this path. 


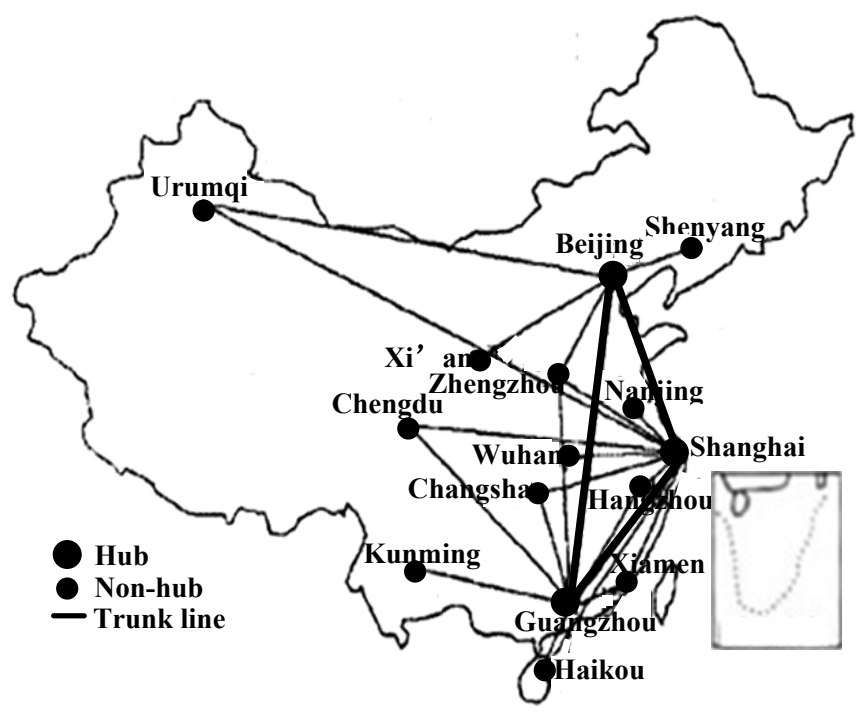

Fig. (2). Uncapacitated $H S$ network $(\mathrm{p}=3, \alpha=0.6, \mathrm{a}=0)$.

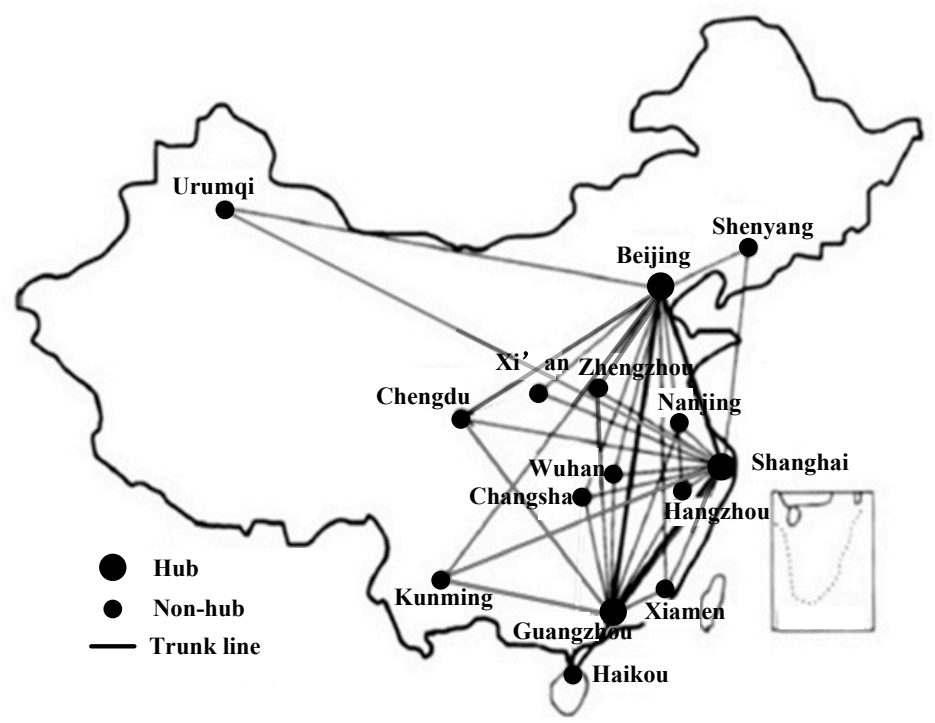

Fig. (3). $H S$ network with congestion cost $(\mathrm{p}=3, \alpha=0.6, \mathrm{a}=1, \mathrm{~b}=2)$.

Table 3. Flow distribution for some paths with three groups of congestion parameters.

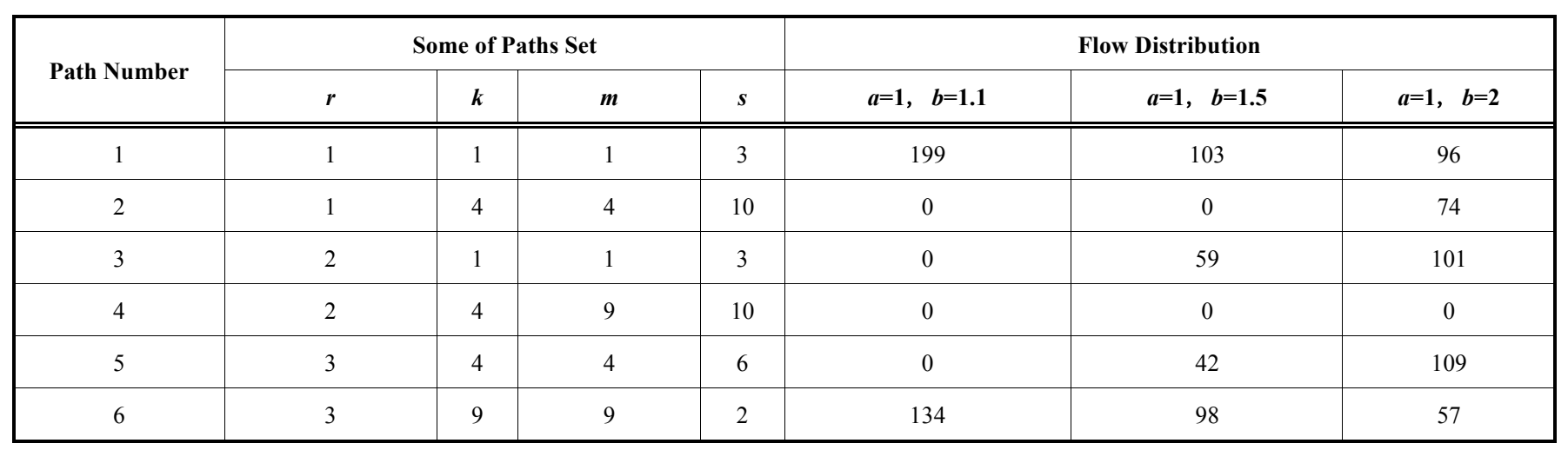


Table 3. contd...

\begin{tabular}{|c|c|c|c|c|c|c|c|}
\hline Path Number & \multicolumn{4}{|c|}{ Some of Paths Set } & \multicolumn{3}{|c|}{ Flow Distribution } \\
\hline 8 & 5 & 4 & 1 & 6 & 0 & 0 & 0 \\
\hline 9 & 5 & 9 & 9 & 15 & 0 & 0 & 0 \\
\hline 11 & 7 & 9 & 9 & 11 & 35 & 50 & 61 \\
\hline 12 & 8 & 9 & 9 & 4 & 61 & 21 & 0.1 \\
\hline 13 & 8 & 4 & 4 & 15 & 186 & 126 & 74 \\
\hline 14 & 10 & 4 & 4 & 6 & 0 & 0 & 0 \\
\hline 17 & 14 & 1 & 9 & 2 & 0 & 0 & 0 \\
\hline 18 & 15 & 4 & 4 & 13 & 0 & 59 & 137 \\
\hline
\end{tabular}

The results show that any hub airport can avoid overloaded traffic flow when considering non-linear congestion cost in the objective function, and the unbalanced flow distribution is reduced between hub airports. For example, As parameter $a$ and $b$ increase, the flow of some paths are changed from zero to a distributed value, such as the $2^{\text {nd }}$ and $3^{\text {rd }}$ path, also the distributed large amount of flow of some paths are diverted, such as $1^{\text {st }}$ and $6^{\text {th }}$ path. Therefore, some busy hub airports can reduce congestion in order to avoid the corresponding flight delay.

\section{CONCLUSION}

This paper proposes CPSO algorithm aimed to HS network design considering congestion cost at hub airports. The objective function is added non-linear congestion cost, when the flow value of hubs increases, the congestion cost is a convex function, which would increase exponentially, therefore, the flow of hub airports can be diverted effectively to minimize the total cost. Comparing with other algorithms of airline network design, $C P S O$ can quickly get the traffic flow distribution of multi paths under specified hubs.

Given each $O D$ demand, $C P S O$ algorithm can get the results of the flow distribution. Before optimization, this algorithm must be determined the set of paths for each $O D$ pair by the exhaustive procedure. Using the method of the mutative scale chaos mutation, which can be very good to accelerate the convergence speed and avoid trapping into local optimization.

Through example demonstration and comparison analysis of Chinese airline transport data, the results show that the algorithm has a good shunt effect on effectively solving hub node congestion problem, and it tries to alleviate the flight delay degree caused by hub node flow increase.

\section{ABOUT THE AUTHORS}

First Author Weiwei Wu, University associate professor of Nanjing University of Aeronautics and Astronautics, Ph.D. The author's major is Management Science and Engineering.

Second Author Hui Wang, master degree in transportation engineering, studying in Nanjing University of Aeronautics and Astronautics. The author's major is Transportation Engineering.

\section{CONFLICT OF INTEREST}

The author confirms that this article content has no conflict of interest.

\section{ACKNOWLEDGEMENTS}

This work was financially supported by the Natural Science Foundation of China (No. 71201081, 71171111).

\section{REFERENCES}

[1] J. Zhu. "Air transportation planning", Northwestern Polytechnical University Press, 2009, pp. 322-364.

[2] M.E. O'Kelly, "A quadratic integer program for the location of interacting hub facilities", European Journal of Operations Research, vol. 32, pp. 393-404, 1987.

[3] T. Aykin, "On a quadratic integer program for the location of interacting hub facilities", European Journal of Operational Research, vol. 46, pp. 409-411,1990

[4] J.F. Campbell, "Hub location and the p-hub median problem". Operations Research, vol. 44, pp. 923-935, 1996.

[5] M.E. O'Kelly, D. Bryan, D.S. Kapov, and J.S. Kapov, "Hub network design with single and multiple allocation: A computational study", Location Science, vol. 4, no. 3, pp. 125-138, 1996.

[6] G.P. Grove, and M.E. O'Kelly, "Hub networks and simulated schedule delay", Papers of the Regional Science Association vol. 59, pp.103-19, 1986. 
[7] H. Yaman, and G. Carello. "Solving the hub location problem with modular link capacities", Computers \& Operations Research, vol. 32 , pp. 3227-45, 2005

[8] V. Mariano, and D. Serra, "Location models for airline hubs behaving as $\mathrm{m} / \mathrm{d} / \mathrm{c}$ queues", Computers \& Operations Research, vol. 30, pp. 983-1003, 2003.

[9] T. Aykin, "Lagrangian relaxation based approaches to capacitated hub-and-spoke network design problem", European Journal of Operational Research, vol 79, pp. 501-23,1994.

[10] J. Ebery, M. Krishnamoorthy, A. Ernst, and N. Boland, "The capacitated multiple allocation hub location problems: formulations and algorithms", European Journal of Operational Research, vol. 120, pp. 614-31, 2000.

[11] J.F. Campbell, G. Stiehr, A.T. Ernst, and M. Krishnamoorthy, "Solving hub arc location problems on a cluster of workstations", Parallel Computing, vol. 29, pp. 555-74, 2003.

[12] I.R. Martin, and J.J. Salazar-Gonalez, "Solving a capacitated hub location problem", European Journal of Operational Research, vol. 184, pp.468-79, 2008.
[13] T.M. Qin, M. Kai, and W.X. Jiang, F.B. Jiang, Z.J. Gang, "OFDM resource allocation algorithm based on chaos particle swarm optimization", Control and Decision, vol. 27, no. 7, pp.1096-1100, 2012.

[14] S. Elhedhli, and F.X. Hu, "Hub-and-spoke network design with congestion", Computers \& Operations Research, vol. 32, pp.16151632, 2005.

[15] J.F. Campbell, "Location and allocation for distribution systems with transshipments and transportation economies of scale", Annals of Operations Research, vol. 40, pp. 77-99, 1992.

[16] R.S. de Camargo, G. Miranda Jr., and R.P.M. Ferreira, and H.P. Luna, "Multiple allocation hub-and-spoke network design under hub congestion", Computers \& Operations Research, vol. 36, pp. 3097-3106, 2009.

[17] J. Kennedy, and R.C. Eberhart, Particle swarm optimization. In: Proceedings of IEEE International Conference on Neural Networks, Piscataway, NJ, 1995, pp. 1942-1948.

[18] D.E. Goldberg, Genetic Algorithms in Search, Optimization, and Machine Learning, MA: Addison Wesley, 1989.

Received: November 11, 2014

(C) Wu and Wang; Licensee Bentham Open

This is an open access article licensed under the terms of the Creative Commons Attribution Non-Commercial License (http://creativecommons.org/licenses/by-nc/3.0/) which permits unrestricted, non-commercial use, distribution and reproduction in any medium, provided the work is properly cited. 